\title{
LA FEMME ET LE DÉVELOPPEMENT AU MAGHREB. UNE APPROCHE SOCIO-CULTURELLE
}

\section{Brahim HACHLOUF}

Oude Hofstraat, 24

2610 Wilrijk

CHAMPS D'INVESTIGATION: linguistiques, culture et problèmes de développement

\section{SUMMARY}

\section{WOMAN AND DEVELOPMENT IN THE MAGHREB. A SOCIO-CULTURAL APPROACH.}

The under-development problem of the Third World can not exclusively be attributed to economic backlogging, political crises or supernatural events. The perseverance of old ancestral values, cultural, social and psychological, still remain obstructions to any social evolution in a country.

The woman's role in society in these countries, is restricted to house-keeping and the bearing of children. A situation which weighs heavy on their economies. This article will shed light on the essential elements which hinder active woman participation in development of their economies, in particular in the Maghrebcountries. Discussed is their personal status, women's position in education and in the work-force.

KEY WORDS: development, emancipation, islam, Maghreb, woman 
"Ce qui caractérise l'occident moderne, c'est d'avoir réussi à masquer sa fascination pour la mort en fascination pour le futur, libérant ainsi les énergies créatrices (...). La différence entre nous et l'occident réside dans la façon dont nous consommons la mort. Le passé. Les occidentaux en font un dessert, et nous essayons d'en faire un plat de résistance."

Fatima Mernissi (sociologue marocaine), Le Harem politique, Albin Michel, 1987, p. 29.

\section{Introduction}

La problématique du sous-développement dans les pays du Tiers-monde ne peut être attribuée au seul retard économique ni à une crise politique ou, simplement, a des facteurs surnaturels. En revanche, la resistance de certaines valeurs ancestrales, d'éléments culturels, sociaux et psychologiques peut être considérée comme une véritable obstruction à tout changement ou évolution sociale dans un pays.

Dans le monde arabe entier la femme occupe une position sociale défavorisée, ce qui rend sa contribution à la production nationale insignifiante, freine ses capacités créatrices au sein des groupements sociaux. Sa fonction est réduite aux travaux ménagers et à la procréation, ce qui débouche sur une croissance démographique galopante qui pèse lourdement sur l'économie de beaucoup de pays du Tiersmonde. Vu le rôle que peut jouer la femme dans la dynamique sociale à travers la planification familiale et sa valeur ajoutée dans l'effort économique, nous avons voulu dans cet essai, repérer certains éléments essentiels qui empêchent une réelle participation des femmes arabes au développement de leur pays. Nous allons particulièrement mettre l'accent sur la femme maghrébine.

\section{La condition de la femme maghrébine: le statut juridi- que ou social}

Pour traiter d'un sujet à caractère social dans le monde arabo-islamique il est habituel chez les sociologues de faire appel à l'histoire de la Nation Arabe dont les fantasmes des mille et une nuits caricaturent les aspects les plus mystérieux des rapports homme/femme. En effet, dans notre analyse il ne s'agit pas de prendre le 
passé pour en faire une projection sur le présent, mais nous croyons que le recours à l'histoire n'est positif que lorsqu'on s'en sert pour faire une autocritique. En ce sens, notre lecture de la culture arabo-musulmane n'est pas une perversion, mais une exploration de la pensée traditionnelle pour en dégager l'image de la femme et la fonction qu'on lui assigne au sein de la société.

Pour nous conformer au thème de notre sujet, posons la question suivante: Pourquoi l'absence de la femme est-elle frappante dans les pays arabes lorsqu'il s'agit du travail rémunéré, de l'activité politique ou même de l'éducation ? Cela est-il dû à la religion, à une politique discriminatoire à l'égard des sexes, ou à des composantes socio-culturelles assez complexes ?

Pour répondre à la première question, ceux qui ont eu l'occasion de connaître la religion musulmane ne s'attardent pas à innocenter l'Islam de tout fatalisme qui aurait comme effet l'interdiction aux femmes de participer au développement de leur pays (1). Au contraire le prophète des musulmans a contracté son premier mariage avec une certaine KHADIJA, femme notable et commerçante de la tribu de Quoraïch; aussi parmi ses autres épouses y avait-il celles qui participaient directement ou indirectement aux batailles tribales entre Mohammed et ses opposants (2). En citant ces épreuves nous n'entendons pas défendre l'Islam comme religion, mais souligner que n'importe quel dogme, pour des raisons diverses, peut être l'objet d'interprétations négatives et parfois volontaires pour parvenir à des fins déterminées. Sinon, par quelle loi religieuse interdît-on aux femmes musulmanes d'accéder au volant (3) ? Dans les pays du Maghreb, même si formellement l'égalité des sexes ne fait pas d'objections, il n'en demeure pas moins que la femme ne bénéficie pas réellement des mêmes droits que les hommes, ne futt-ce qu'au niveau de sa participation politique et au travail salarié, par contre la mobilisation des masses pour stimuler la production et la productivité, oblige à ne pas épargner l'énergie des femmes. Revenons à la condition de la femme du point de vue religieux et social pour constater que la préminence masculine est significative en Islam, mais le Coran ne prive la femme d'aucun droit fondamental dans le sens strict du droit positif. Son droit à la vie est assuré par le verset I de la sourate des femmes (4), mettant ainsi fin à la pratique pré-islamique de l'enterrement des filles vivantes à leur naissance. Le droit au travail est également assuré par le fait que les croyants et les croyantes ont les mêmes devoirs devant Dieu. Le droit à l'éducation est beaucoup plus recommandé pour les deux sexes: "L'Islam exhorte le musulman, homme ou femme, à s'instruire du berceau à la tombe", a dit le prophète (5). La protection de la femme dans les rapports matrimoniaux est singulièrement garantie par le Coran; la première source de la jurisprudence musulmane. Dieu Y consacre une sourate aux femmes. Leur droit à l'héritage, leur part dans des cas différents et leurs droits à l'égard de leurs maris 
Tableau 1:

Taux effectif de l'éducation de la femme dans les trois pays du Maghreb

\begin{tabular}{|c|c|c|c|}
\hline \multicolumn{4}{|l|}{$\begin{array}{l}\text { Indicateurs de l'enseignement des } \\
\text { femmes }\end{array}$} \\
\hline PAYS & ALGERIE & MAROC & TUNISIE \\
\hline \multicolumn{4}{|c|}{$\begin{array}{l}\text { Nombre d'inscriptions dans } \\
\text { l'enseignement en \% du groupe d'åge } \\
\text { pertinent } 1986\end{array}$} \\
\hline - primaire & 85 & 62 & $108^{*}$ \\
\hline - secondaire & 45 & 27 & 33 \\
\hline \multicolumn{4}{|l|}{$\begin{array}{l}\text { Nombre de femmes } \\
\text { pour } 100 \text { hommes }\end{array}$} \\
\hline - dans le primaire & 79 & 62 & 80 \\
\hline - dans le secondaire & 72 & 67 & 71 \\
\hline $\begin{array}{l}\text { Pourcentage des femmes adultes } \\
\text { analphabetes } 1989\end{array}$ & 63 & 78 & 59 \\
\hline$\%$ total femmes analphabètes & 50 & 67 & 46 \\
\hline
\end{tabular}

Source: Rapports de la Banque Mondiale sur le développement dans le monde 19881990.

* Le chiffre de $108 \%$ pour la Tunesie signifie que même quelques filles qui n'ont pas encore atteint l'âge de la scolarisation sont scolarisées. 
y sont explicités. Même si la polygynie est autorisée, elle est conditionnée par l'équité à l'égard de chacune des épouses. Certains penseurs musulmans voient dans cette condition une pure interdiction car le polygame ne peut pas être équitable, au moins sur le niveau sentimental, envers plusieurs épouses (voir dans ce sujet Mohammed AZIZ LAHBABI, Le personnalisme musulman, PUF 1967, p. 78). Dans ce sens la Tunisie a aboli la polygynie, au Maroc et dans d'autres pays arabes la femme peut annexer à l'acte du mariage des articles par lesquels le mari s'engage à respecter la monogamie et en cas de divorce à lui payer les indemnités (M.A. LAHBABI, op.cit. p. 75).

Si l'attitude de l'Islam est sans équivoque à l'égard de la femme, celle de certains théologiens musulmans differe lorsqu'ils réclament le retour aux sources de l'Islam comme cela à été révélé au 7 siècle. Car c'est un retour vers un passé réduit et sélectionné pour y lire le portrait d'une femme voilée, cloîtrée et dépossédée de tout pouvoir de s'imposer dans la société.

C'est ce portrait obscur qui tempère le comportement de l'homme vis-à-vis de sa partenaire: la femme. Dès lors l'ouverture de la femme sur le monde extérieur devient une transgression aux normes tracées par une passé fantôme imaginé et nos assimilé.

Dans les trois pays de Maghreb, les codes de statut personnel élaborés après l'indépendance permettent tous à la femme d'accéder à l'enseignement et au travail, tout comme l'homme(6). Au Maroc, l'article 8 de la Constitution du 10 mars 1972 stipule que "L'homme et la femme jouissent de droits politiques égaux. Sont électeurs tous les citoyens majeurs des deux sexes jouissant de leurs droits civils et politiques" (7). Cependant, dans la pratique, en raison des contraintes familiales (8), la femme ne jouit que des droits voulus par le chef de famille et non pas de ceux édictés par les institutions législatives. Nous allons illustrer cela par le cas de l'enseignement.

\section{La femme et l'enseignement}

Une simple lecture des statistiques publiées par les rapports annuels de la Banque Mondiale, de l'UNESCO ou d'autres organisations internationales nous permet de nous rendre compte du degré de marginalisation des femmes maghrébines. En effet, à l'exception de la Tunisie, le taux d'alphabétisation des femmes est assez médiocre par rapport aux autres pays du même niveau de développement et cela après des dizaines d'années d'indépendance.

Il ressort des chiffres du tableau 1 que l'effort consenti par les Etats du Maghreb afin d'insérer la femme dans le processus de développement social est insuffisant. 
Cette insuffisance devient plus éclatante lorsqu'on tient compte des femmes alphabétisées année par année depuis l'indépendance. Le taux des femmes âgées illettrées dépasse $95 \%$. Quelles sont les raisons de ces résultats assez médiocres dans des pays qui disposent des moyens pour généraliser l'enseignement et accélérer l'évolution de leurs pays ? En réponse à cette question il faut dire tout d'abord que le premier obstacle à l'instruction des femmes est inhérent à l'inconscience des citoyens eux-mêmes. En effet, les populations arabo-berbères maghrébines ont hérité des traditions et coutumes assez complexes à travers divers contacts avec les populations du bassin méditerranéen, du Moyen-Orient et de l'Afrique noire. C'est pourquoi la scolarisation des filles se heurte à une conception mystérieuse de la féminité qui est associée à certaines valeurs confuses comme l'honneur, la sexualité, la virilité et le prestige au sein du lignage. Cela se traduit du côté des parents, par une réticence ou un refus pur et simple de scolariser leurs filles, redoutant l'ouverture de celle-ci au monde et les "conséquences" qui peuvent en résulter. dans d'autres cas assez semblables certains chefs de familles se pressent de retirer leurs filles des écoles dès que celles-ci deviennent pubères (9).

Quant à l'indifférence des responsables des pays concernés à l'égard de cet état de choses, elle peut être justifiée par le maintien du statu quo. Car tant qu'une partie de la population, à savoir les femmes, est mise à l'ecart, cela les arrange et les conforte dans leurs positions. Voilà pourquoi, depuis l'indépendance, on assiste à un lent recul du taux d'analphabétisme chez les femmes. Par exemple entre 1971 et 1982 le pourcentage de l'analphabétisme au Maroc est passé de 66,4 à 57,8 \% chez les hommes et de 90,2 à $82,8 \%$ chez les femmes (10). Même constat pour l'Algérie et aussi la Tunisie, le pays modèle dans la région, en ce qui concerne la conscientisation de la femme où entre 1975 et 1984, le pourcentage des analphabètes est passé de $48,9 \%$ à $39,5 \%$ pour les hommes et de 75 à $59 \%$ pour les femmes (11).

Les répercussions de l'état d'ignorance que vit la plus grande proportion des femmes maghrébines sont aisément repérables. Il suffit de consulter les rapports du Bureau International du Travail pour constater les faibles effectifs féminins dans tous les secteurs de l'économie. Il suffit également de jeter un regard sur la situation démographique pour se rendre compte de la machine procréatrice qu'est la femme dans cette région. Nous allons revenir sur ces deux thèmes, mais tout d'abord il faut signaler qu'il existe un rapport assez étroit entre le degré de conscientisation d'une femme d'un côté et sa participation à la vie politique de l'autre. C'est une des raisons pour laquelle le pouvoir, dans les pays maghrébins, et les hautes fonctions de responsabilité se concentrent toujours entre les mains des hommes, balayant ainsi les principes de l'égalité et de la démocratie car: "Les droits politiques accordés à la femme font partie de la logique démocratique. 
Constituant une force du potentiel humain et du corps électoral, la femme ne doit pas être marginalisée (...) elle a des services à rendre à la société et des intérêts à défendre" (12). Il faut noter qu'à part un poste ministériel en Algérie et un autre en Tunisie, la présence de la femme au sein des gouvernements maghrébines et des institutions politiques est quasi nulle.

\section{La réalité démographique: quelle issue ?}

Nul ne conteste que le rythme de la croissance démographique dans les pays du Tiers-Monde se caractérise par la rapidité. On en déduit certes, une baisse du taux de mortalité due à une amélioration graduelle des conditions sanitaires, mais en l'absence d'une croissance économique parallèle, le phénomène de l'explosion démographique est, aujourd'hui, un des défis les plus pressants pour les pays en voie de développement. Dans ce contexte nous assistons, après la deuxième guerre mondiale, dans les pays du Maghreb, à une croissance démographique spectaculaire. La population est passée de 21 millions en 1950 (12) à environ 57 millions actuellement (14), c'est-à-dire qu'elle a triplé dans un intervalle de 40 ans.

Cette croissance démographique vertigineuse devient assez inquiétante à partir du moment ou nous diagnostiquons la réalité économique et sociale de ces pays. en effet avec une croissance économique en régression (15), (le taux annuel moyen de croissance en \% du PIB était entre 1965 et 1980 de 5,6 pour le Maroc, 6,8 pour l'Algérie et de 6,6 pour la Tunisie entre 1980 et 1988 était respectivement 4,2, 3,5 et 3,4 ), un taux de chômage (non indemnisé) assez élevé et une dette extérieure qui pèse lourd sur l'économie (16), les dépenses publiques se réduisent de plus en plus et les Etats se trouvent ainsi incapables de répondre aux besoins de leurs populations en matière d'éducation, de santé, d'habitat et de création d'emplois. Selon les estimations de l'ONU le taux de fécondité dans les pays du Maghreb ne rejoindra celui des pays développés qu'après l'an 2015 (17). On pourrait imaginer, à la lumière de la conjoncture économique actuelle, les problèmes socio-économiques qui pourraient découler de la surpopulation de ces pays. on pense notamment à la sécurité alimentaire dans la région et à l'équilibre entre les besoins de la population et les ressources naturelles dont elle dispose. Les responsables de ces pays doivent agir vigoureusement et s'attaquer aux origines de la croissance démographique vertigineuse. Il semble que la femme, de par sa fonction au sein de la famille et sa condition sociale, peut être le frein ou l'accélérateur de cette croissance et elle peut jouer un rôle déterminant dans la tendance démographique de n'importe quel pays. Mais comment ? Prenons l'exemple de la fécondité. Celleci est conditionnée par deux types d'éléments: 
Schéma 1: La dépendance du taux de fécondité de la condition de la femme

Relation de dépendance

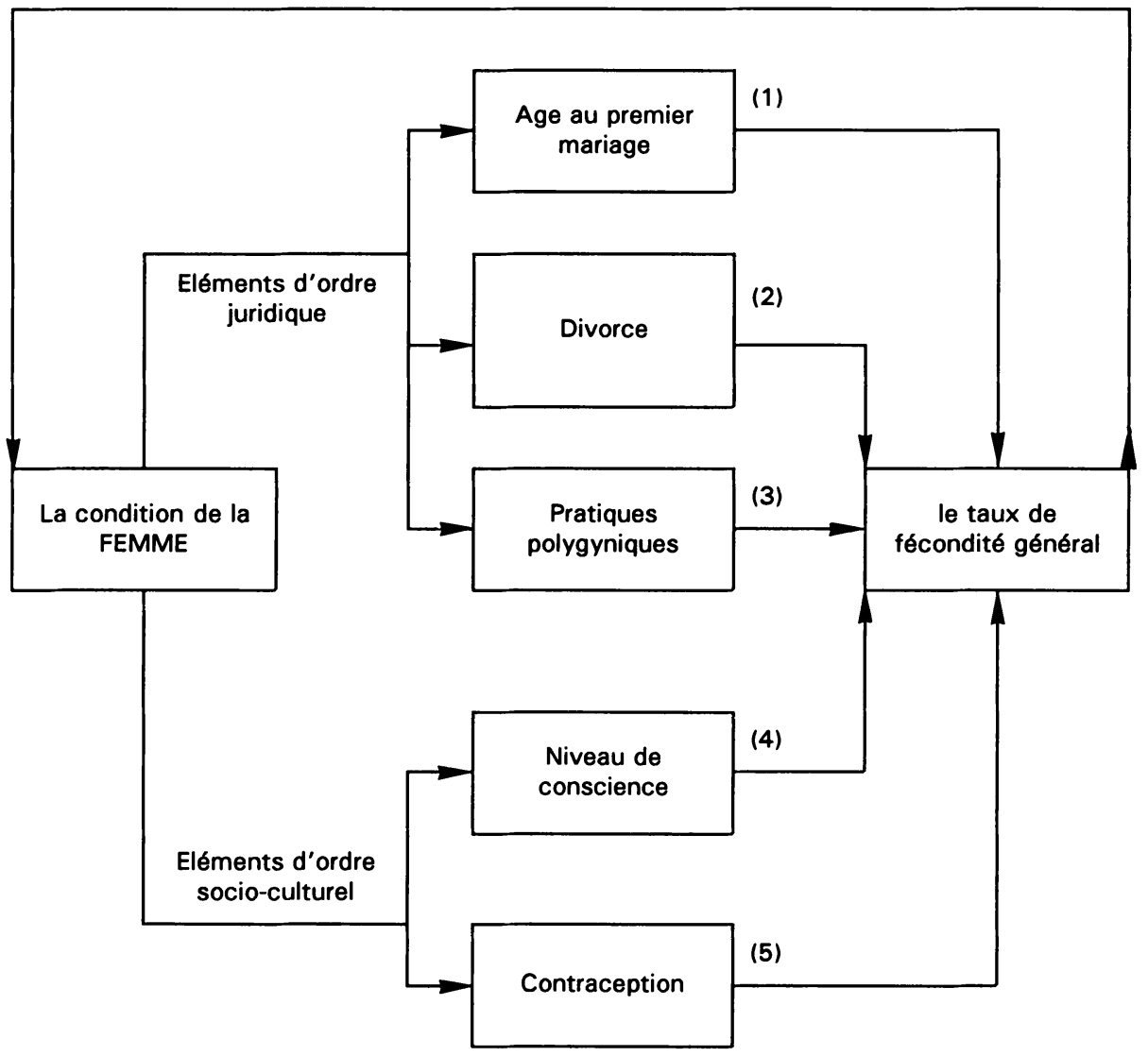

(1) "plus il est bas plus haut est le taux de fécondité général"

(2) "plus il y a de divorce plus élevé est le taux de fécondité général"

(3) "plus elles sont fréquentes plus est le taux de fécondité général"

(4) "plus il est élevé moins est le taux de fécondité général"

(5) "plus elle est utilisée moins est le taux de fécondité général"

Note: Les pratiques polygyniques ont pour but en principe d'accroitre le nombre d'enfants surtout dans les familles rurales, mais elles peuvent être un facteur négatif à la fécondité générale dans le cas d'un époux stéril. 
- le premier d'ordre juridique, concerne la réglementation des rapports matrimoniaux comme la fixation de l'âge au premier mariage et les contraintes imposées sur le divorce et la polygynie;

- le deuxième est relatif à la condition personnelle de la femme, c'est-à-dire à son niveau de conscience, son ouverture sur le monde extérieur et son attitude vis-àvis des moyens contraceptifs.

C'est ce que nous pouvons montrer en schéma 1.

La femme alors se présente, dans ce schéma, comme un carrefour ou se croise toute une série de facteurs déterminant l'essor démographique d'un pays. D'où, on peut déduire sans risque d'erreur que la maîtrise de la croissance démographique repose sur l'amélioration de la situation de la femme. Il est certain que le retard d'âge au premier mariage et la mise sur pied, par les pays concernés, des mesures persuasives à l'égard des pratiques polygyniques et le divorce (18) injustifié. Tout cela mène à un déclin de la fécondité. Car dans les pays musulmans le mariage est le cadre exclusif de la procréation. Et si la femme comprend l'utilité de la contraception l'organisation de la famille et de la société en général deviendra plus qu'évidente.

Par ailleurs, au Maroc et en Algérie, quoi que l'âge minimum au premier mariage pour les filles est fixé à 16 ans (19), ce barrage est souvent brisé surtout dans les milieux ruraux.

L'Algérie est un des pays arabes les plus féconds. En 1985 le nombre d'enfants par famille était de 6,1 (20) et seulement $7 \%$ des femmes mariées en âge de procréer utilisent les méthodes artificielles de contraception contre 27\% du Maroc et $42 \%$ en Tunisie (21). La réduction du taux de croissance démographique d'un pays dépend également de la volonté de ses responsables de mettre en oeuvre une politique rigoureuse de planning familial et de prendre des mesures répressives à l'égard des familles nombreuses. Or cette volonté, paraît-il, fait défaut aux responsables algériens qui déclaraient en 1974 à la conférence mondiale sur la population à Bucarest: "Le meilleur contraceptif c'est le développement" (22). Cette devise résume en quelque sorte la philosophie du développement chez certains dirigeants maghrébins: le développement économique permet le ralentissement de la croissance démographique, alors qu'on pourrait débuter par cette dernière pour espérer un développement global, c'est-à-dire un développement économique et social.Dans l'immédiat le problème de la démographie ne peut être abordé que si on améliore la condition de la femme dans toutes les régions du 


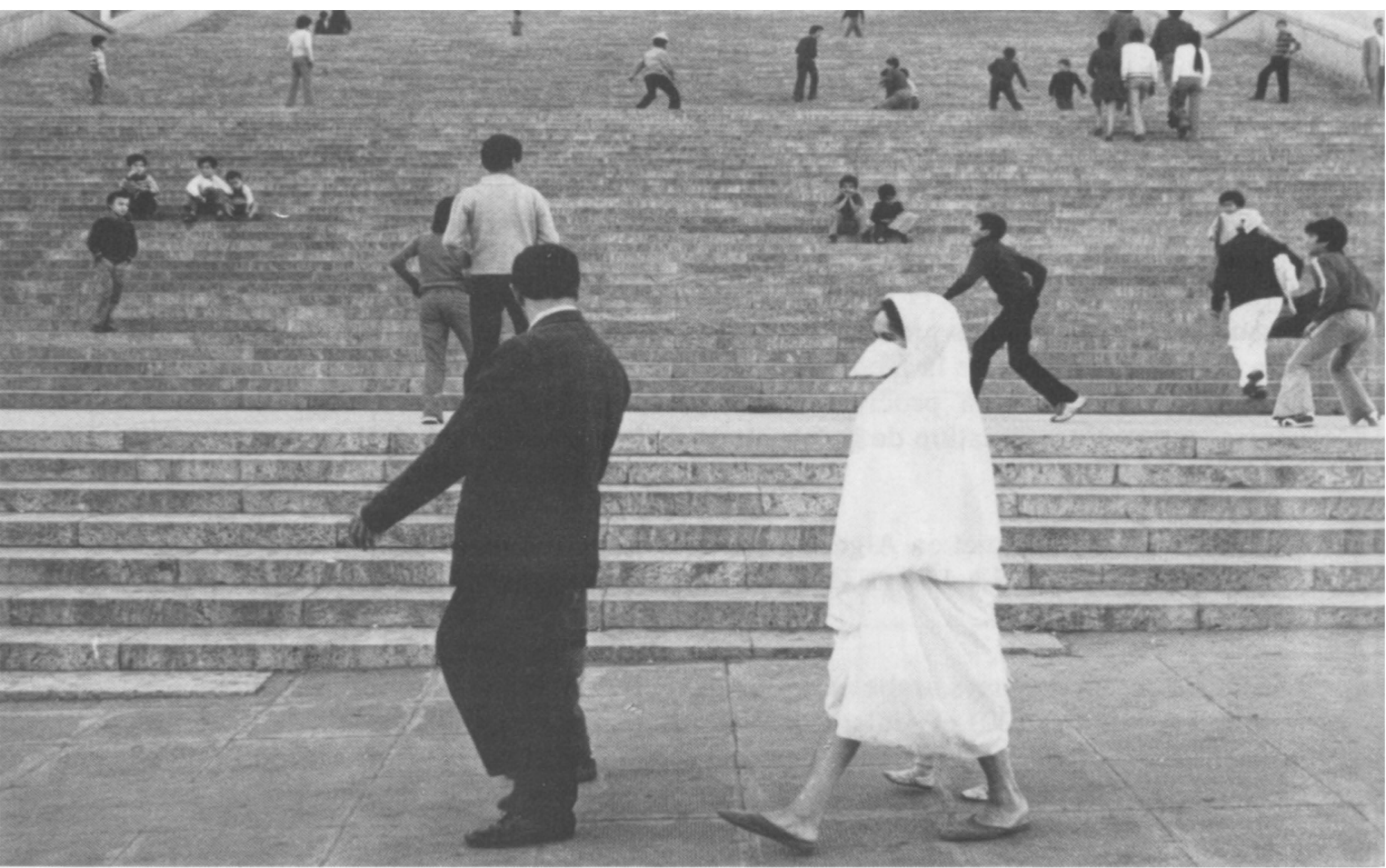

A Alger

D.Beke ${ }^{\ominus}$ 
Maghreb. A ce sujet, il faut généraliser l'éducation et la rendre obligatoire tant dans les milieux ruraux que dans les milieux urbains, intensifier la conscientisation de la population et mobiliser la femme de manière permanente. Toutes ces mesures doivent constituer les démarches préalables à une modernisation sociale qui aboutira à une modernisation économique. Car "les économistes s'accordent à penser que les efforts et les progrès enregistrés dans la lutte contre le sousdéveloppement sont anéantis au fur et à mesure par l'explosion démographique"(23).

\section{La femme maghrébine et le travail}

L'évaluation de la contribution des femmes à l'économie nationale au Maghreb est difficile à effectuer pour au moins deux raisons fondamentales:

- premièrement le caractère informel et saisonnier des activités exercées par cellesci et sa clandestinité parfois (24) rend douteuses toutes enquêtes ou statistiques relatives à ce sujet;

- deuxièmement la notion d'activité, même, telle qu'elle est forgée par le bureau international du travail ne représente pas un instrument de mesure approprié. Parce que l'organisation en question exclut de ses comptes l'effort des femmes dans les travaux familiaux, et leurs activités à la campagne, parfois pénibles, comme le transport de l'eau, du bois et les travaux à domicile qui offrent des exemples de rentabilité importants. Néanmoins il faut reconnaître que la participation des femmes maghrébines à l'activité économique hors du foyer est très limitée par rapport à d'autres pays du Tiers-Monde. En terme de travail rémunéré, le taux des actives de 15 à 65 ans est de $9 \%$ pour l'Algérie 17,6\% pour le Maroc et $23 \%$ pour la Tunisie selon les recensements respectifs de 1985-1982 et 1984 publiés par l'OIT (25). A titre de comparaison la contribution des femmes actives africaines à la production alimentaire au sud du Sahara est de $90 \%$ (26).

Comment peut-on expliquer ce phénomène ? Pour répondre à cette question il faut puiser dans la culture arabo-berbère qui est le cadre représentatif de l'identité des individus ou des groupes d'individus. En d'autres termes il faut explorer la "mémoire populaire", les coutumes des gens et leur vision du monde. Un diction Algérien nous apprend que: "La femme ne sort que trois fois dans sa vie: une fois du ventre de sa mère, une seconde fois pour se rendre chez son mari et une troisième fois pour se rendre au cimetière" (27). C'est à travers cette image symbolique et anecdotique que l'on se fait de la femme qu'il faut dégager la fonction que l'on lui assigne dans une telle société régie par le système patriarcal, basé sur la misogynie et orchestré par des traditions archaïques et assez complexes. La division sexuelle du travail instituée par ces traditions confere à la femme 


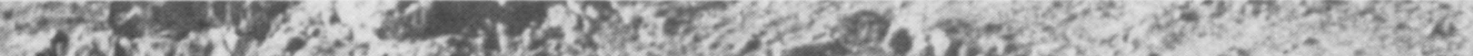

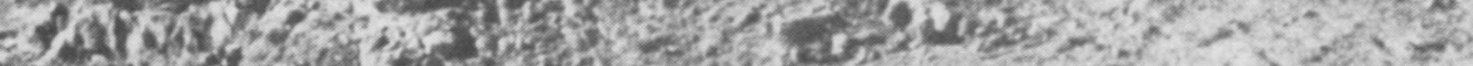

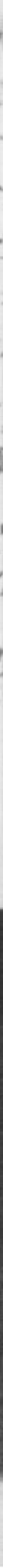

Femme targuia dans le Hoggar (Algérie)

D. Beke ${ }^{\circ}$ 
l'entretien du foyer, la procréation des enfants mâles et leur éducation. Tandis que l'homme se charge d'assurer les besoins vivriers et matériels de ce foyer. Dans la réalité ce partage traditionnel des tâches repose sur un cliché stéréotypé chaque fois que l'on évoque la femme. Celle-ci est indispensable, bien sûr, pour satisfaire quelques besoins instinctifs de l'homme, mais elle est un diable à craindre. C'est elle qui a plus tendance à commettre les péchés. Par ses ruses, son art de séduire, elle est dans la culture arabo-islamique la "FITNA", c'est-à-dire la créatrice du désordre et des tensions au sein du groupe. C'est dans ce symbolisme que le contact homme/femme bouleverse les normes ancestrales et la mixité à l'école, au travail et dans les lieux publics perturbe le schéma social habitué; parce que la femme se situe dans un contexte ou le sexuel, le moral, le religieux et le traditionnel sont tous indissociables. Et c'est pour cela que son intégration dans les secteurs de production modernes est loin d'être réalisée malgré l'influence de la culture occidentale et les exigences de la modernisation sociale qu'impose notre époque.

\section{La femme et l'imaginaire}

Si la relation entre la modernité de la femme et le développement économique et social est déterminée à la fois par des concepts scientifiques (indicateurs de développement) et mythologiques (indicateurs moraux et métaphysiques), cette relation prend des dimensions complexes à partir du moment où on pénètre dans la culture des pays maghrébins, et par culture nous entendons:

"Cette totalité où entrent les ustensiles et les biens de consommation, les chartes organiques règlants les divers groupements sociaux, les idées et les arts, les croyances et les coutumes" (28).

Car les idées, les croyances et les coutumes paraissent en large mesure déterminer le sort des femmes maghrébines. Nous allons essayer d'illustrer cela à travers quelques exemples.

\subsection{Le voile}

A l'instar de beaucoup de pays musulmans le port du voile par les femmes au Maghreb était toujours pour les occidentaux, signe de naïveté, d'exotisme du sousdéveloppement et de l'oppression religieuse. Pourtant la religion seule ne paraît pas l'élément qui explique amplement le caractère socio-culturel de l'habit dans n'importe quelle société. Car le comportement vestimentaire peut exprimer une 


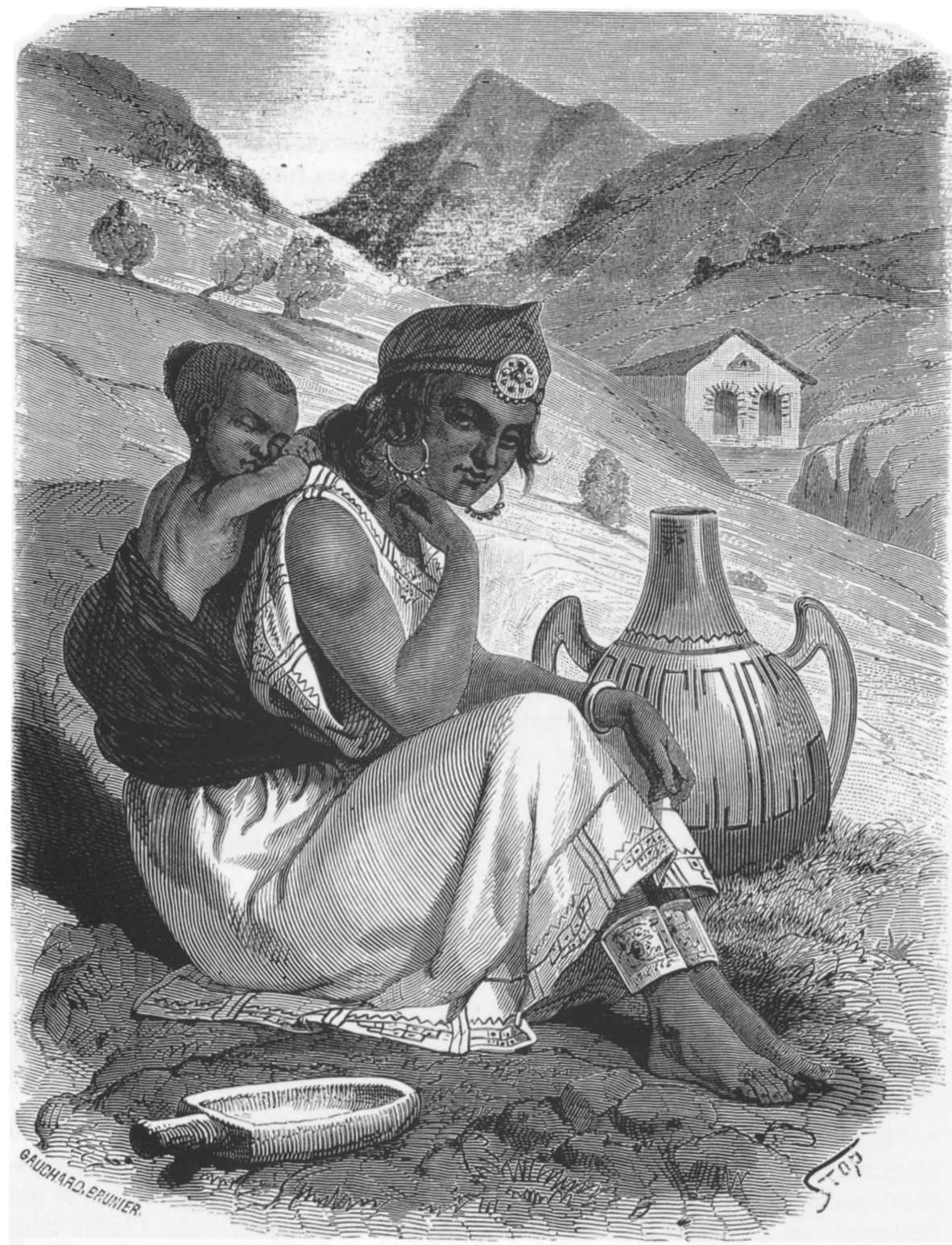

Femme kabyle. - Dessin de Stop d'après un croquis du commandant Duhousset.

Gravure dans:

Commandant DUHOUSSET, Excursion dans la Grande Kabylie. Notes et croquis recueillis entre la Méditerranée et le Djurdjura. In: Le Tour du Monde. 1867 deuxieme semestre. Paris, Hachette, 1867 
attitude culturelle, un caractère économique, une situation sociale ou une dimension relationnelle (29), et il peut varier en forme et en contenu selon les pays et les périodes historiques. Le Tchador Iranien, le hijab Islamique et le foulard maghrébin n'ont pas la même connotation dans la culture arabo-islamique, quoique les occidentaux classent tous sous la même étiquette. Bien avant l'Islam, tout comme après, des femmes musulmanes du Proche-Orient et chrétiennes, se sont toujours couvert le visage d'une voile noire, qui semble être hérité des Byzances (30). Au Maghreb, avant que les deux cultures arabe et berbère se superposent, dans les sociétés rurales et berbères, les Kabyles, les Maures, les Touaregs, les femmes avaient le visage découvert (31) avant que celles-ci cèdent au phénomène de l'acculturation et portent le voile sous une raison ou une autre. Le voile comme il était exprimé dans les sociétés maghrébines, était symbole d'une classe sociale élevée (32), il fut pour une longue période un privilège qui distingue la femme citadine de la femme rurale. La preuve est que les paysannes marocaines se voilent en quittant la campagne jusqu'à l'acquisition personnelle ou dans leur descendance féminine d'une assez réelle urbanité (33). Le but des citadines de porter le voile était de conserver la frâicheur de leur teint, tandis que pour les bédouines il devait leur servir à se protéger du soleil et du sable du désert, où même les hommes se voilent (34). Voilà en quelque sorte la dénotation ancienne du voile. Mais le voile va devenir un symbole d'isolement et d'écartement de la femme de la vie active, à partir du moment où la condition féminine a suivi la courbe de l'apogée et de la décadence islamique. Le pire est qu'à partir de la confrontation des sociétés arabomusulmanes avec l'occident, à travers la colonisation, la femme deviendrait un des moyens pour défier l'occident: le repli sur soi, le voile, la claustration de la femme et son obligation à se courber aux coutumes ancestrales, sont devenus dès lors des expressions d'un refus pour l'idéologie occidentale. On perçoit la femme comme gardienne des valeurs "sacrées" et comme protectrice du patrimoine. Le choc culturel qu'ont subi les sociétés colonisées a, certes, modifié le contenu de beaucoup de valeurs et d'aspects culturels au nord de l'Afrique, mais la politique des pays du Maghreb indépendant à l'égard de la femme pouvaient jouer un rôle prépondérant dans la continuation à voiler la femme ou à lui ouvrir les horizons qui lui permettent non pas de défier l'occident en se recroquevillant et en s'isolant, mais le défier par la concurrence au savoir scientifique et par le travail rentable. Car de nos jours le port du voile est devenu signe du fatalisme et de régression et des idéologies orthodoxes (35), et non pas un aspect simplement culturel. 


\subsection{L'exotisme: sexualité et tabous dans la société maghrébine}

\subsubsection{La sexualité}

La plupart des recherches liées au développement au Maghreb ou du monde arabe en général, dans le domaine des sciences sociales ont été concentrées sur les thèmes économiques et politiques sans pour autant accorder aux problèmes de la famille et de la femme l'intérêt qu'il leur faut. Alors que les études sociologiques et anthropologiques peuvent donner un coup de pouce aux transformations sociales et à la libération de la femme par l'analyse de la dynamique des groupes sociaux et par une "archéologie" dans leur système de pensée.

Dans notre sujet le rapport entre la femme et la sexualité reflète une relation conflictuelle entre la tradition et la religion, d'une part, qui définissent le statut de la sexualité dans un cadre astreint du mariage légal "licite", et la modernité, d'une autre part, où la liberté sexuelle de la femme est synonyme de son émancipation.

"Tradition et progrès sont les deux plus grands ennemis de nos sociétés. Mais ce qui frappe c'est qu'en matière d'éthique la modernité ne peut être qu'une volonté du non. Non à la négation de la femme, non aux divers interdits qui frappent la sexualité. Ce n'est pas par hasard si la modernité a, dès le départ, été synonyme d'émancipation féminine et qu'elle a fini aujourd'hui par être synonyme d'émancipation sexuelle" (36).

La sexualité est donc comme le voile, une phénomène sociologique qui caractérise la crise d'une société maghrébine en transition où l'irruption de la femme met en danger tout un système de valeurs; toute une religion qui dans ce cadre reconnait implicitement la suprématie de l'homme par l'autorisation de la polygynie et l'interdiction de la polyandrie.

A vrai dire les femmes, aujourd'hui, conscientes de leur rôle dans la dynamique du développement ne veulent plus être ce champ qu'on cultive (37), mais elles veulent être des cultivatrices dans le champ. C'est-à-dire qu'elles veulent être des agents économiques reconnus par la société et jouissent des mêmes droits que l'homme.

Le mythe sexuel chez la femme maghrébine se traduit par un ensemble de tabous, créé soit par la tradition, soit par la religion, mais que la société maghrébine moderne continue à considérer comme des valeurs sacrées et comme caractéristique de l'identité culturelle. Nous allons revenir plus loin sur un de ces tabous, mais tout d'abord il faut se poser la question: comment le sacré et le mythe sexuel 
constituent-ils des freins pour le développement au Maghreb? Nous répondons qu'ils sont des freins au développement lorsque les parents retirent leurs filles des écoles dès que celles-ci deviennent pubères, et les empêchent de travailler dans des milieux mixtes et les obligent à se draper pour cacher leur féminité. Ici nous serons confrontés, encore, à une autre question: Pourquoi la sexualité est-elle entourée d'assez d'énigmes chez les populations maghrébines ? La réponse à cette question réside, incontestablement, dans l'image que les maghrébins ont de la femme. Cette image, qui fait partie intégrante de la culture arabo-berbère, associe la femme à un objet de plaisir et qu'au cas où elle jouit d'une grande liberté, elle devient source de malaise pour l'homme, d'autant plus que le mot arabe "FITNA" (38) auquel elle est assimilé, signifie rébellion, conspiration et anarchie. De là il faut réprimer la femme et cette répression commence par la sexualité pour se transformer à l'interdiction de tout contact avec le monde extérieur: le travail, l'école, les gens ... (rappelons-nous le dicton algérien cité antérieurement).

Par ailleurs, le principe de l'égalité des sexes revendiqué par les femmes et qui fait partie intégrante de l'idéologie moderniste, est combattu au nom de la religion et de la morale.

Nous ne croyons pas pouvoir délimiter ici toute la problématique de la sexualité au Maghreb dans sa relation avec la modernité sociale parce que celle-ci fait partie d'une culture toute entière, fertile pour les recherches sociologiques et anthropologiques, mais nous croyons que l'exemple suivant explicite mieux cette problématique.

\subsubsection{Le tabou de la virginité}

Dans la société maghrébine où les relations sexuelles ne sont pas permises hors du cadre légal du mariage, la forme le plus extrême de la répression de la femme se manifeste, consciemment ou inconsciemment, dans les rites qui entourent la virginité la nuit des noces. En effet la fine membrane dont disposent les filles vierges est la cause de beaucoup de perturbations sociales. L'hymen est l'honneur des familles puisque le mariage n'est pas l'union de deux êtres, mais toujours celle de deux familles (39). Quand la paroi vaginale ne saigne pas la nuit de noces, l'hymen devient générateur de drames souvent lamentables (40), alors qu'il est confirmé médicalement que dans plus de $30 \%$ des cas les femmes, physiologiquement, ne sont pas dotées d'un hymen (41); dans ce cas la défloration n'entraîne pas de saignement. Ces traditions qui constituent de véritables contraintes psychologiques sur la vie privée de la femme, freinent chez elle toutes les facultés de penser, et d'agir, et transforment son être en un monde de mythologie et d'érotisme. Par exemple c'est pour combattre le mythe de la virginité que la femme maghrébine 


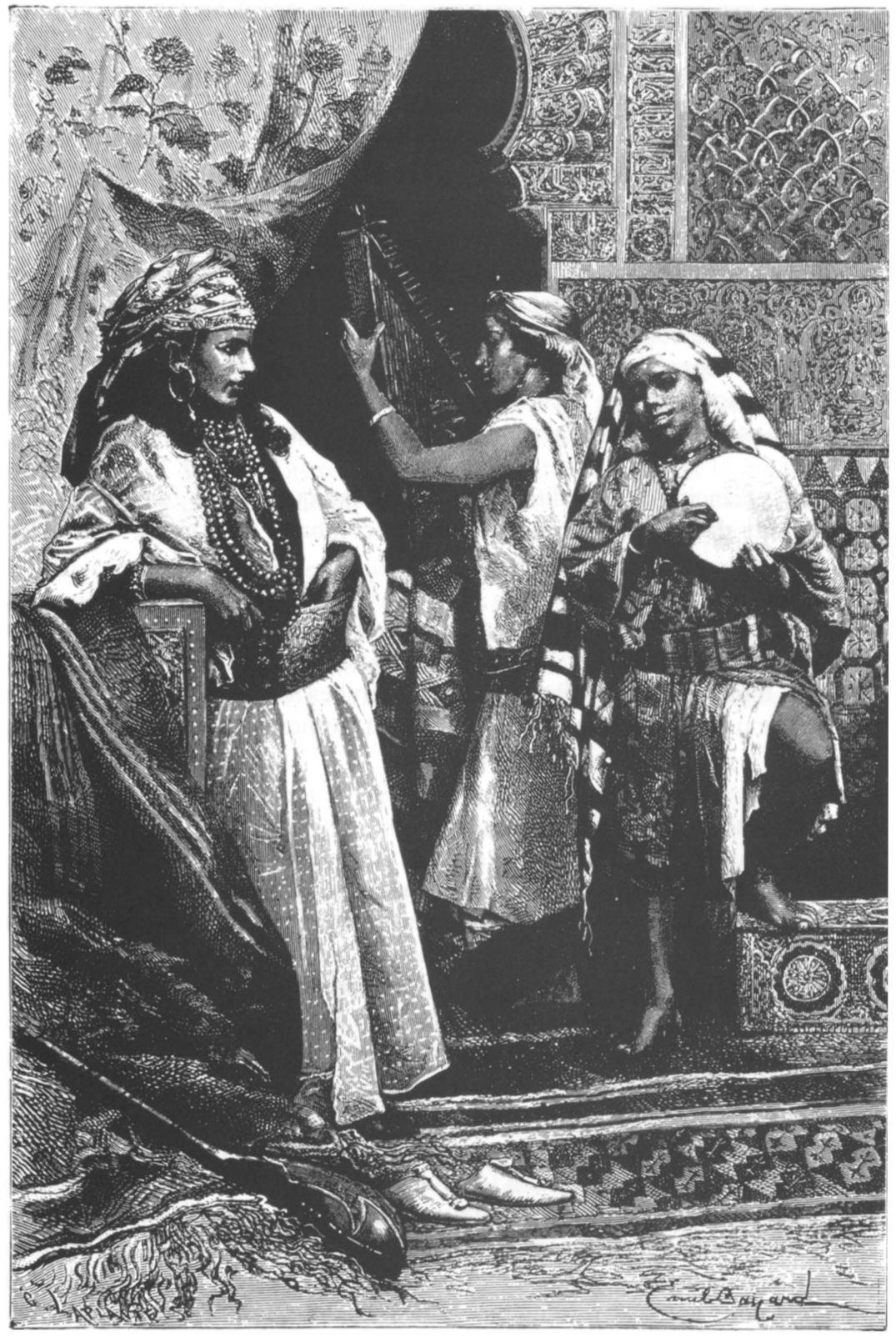

Musiciennes marocaines

Gravure des années 1850 
fait recours à la magie ou à des opérations chirurgicales pour réparer la "honte" et sauver "l'honneur" de sa famille. Ici on se pose, encore, une autre question: Est-ce que ce n'est pas du sous-développement lorsque toute une industrie de "replâtrage" (42) des virginités se développe dans les cliniques des capitales maghrébines et sous l'approbation implicite des autorités qui n'entendent pas intervenir, sur le plan de la morale individuelle ? Une autre fois en l'absence d'une politique cohérente à l'égard des tabous (43), c'est la tradition qui va répondre par la négation. Parce qu'il est ancré dans la mémoire de l'homme que la femme est ce trésor qui sauvegarde l'honneur. La croyance à la virginité dans la morale sexuelle est toute imprégnée d'intérêt de propriété. La femme devient une chose appartenant à un lignage. La virginité accentue la chosification de la fille et la réduit à un hymen non perforé (44), sa valeur marchande, son honneur sont fonction de la préservation de cette chose précieuse.

Encore faut-il ajouter que l'image de la propriété de la femme dans la société maghrébine est d'autant plus reflétée dans les formes des mariages des différents tribus et lignages (on ne fait pas la distinction ici entre zones rurale et urbaine). La psychiatre marocaine Ghita EL KHIYAT BENNAI constate que contrairement aux systèmes européens, l'exogamie chez les arabes est grandement déshonorée parce que d'après leur conception, les preneurs de femmes sont supérieurs aux donneurs. L'auteur écrit:

"On épouse sa cousine germaine en ligne paternelle (Bint El Am ou fille de l'oncle). Contrairement aux systèmes européens (...) Là apparaît donc cette étrange conception de l'honneur parce qu'il s'inscrit dans un sentiment suraigu de jalousie: on ne donne pas ce qui est à soi - les femmes du lignage - aux autres" (34).

Un autre maghrébin dévoile la conception sur laquelle est basée l'endogamie au Maghreb. Il cite:

"L'assise économique de l'endogamie systématisant la pratique du cousinat freinant par là même la rotation des femmes, l'expansion et l'expression de tout amour qui n'est pas conforme aux intérêts du groupe, tout cela ne pouvait qu'aboutir le plus naturellement du monde à un esclavage à peine déguisé de la femme arabe" (45).

Si le mariage endogame repose sur une telle conception, sa forte fréquence dans les milieux maghrébins et surtout chez les berbères, suscite beaucoup d'interrogations autour de l'émancipation de la femme maghrébine. 


\section{Tableau 2}

Tableau comparatif de différents types de relation existant dans quelques mariages berbères

\begin{tabular}{lccccc|}
\hline & Touaregs & Ahasas & Rifains & Kabyles & Aït Jrrar \\
$\begin{array}{l}\text { Mariages endogames } \\
\text { cousine parallele } \\
\text { patrilinéaire }\end{array}$ & 0 & 0 & + & + & + \\
$\begin{array}{l}\text { Mariage exogame y } \\
\text { compris avec la fille de } \\
\text { I'oncle maternel }\end{array}$ & + & + & 0 & 0 & 0 \\
$\begin{array}{l}\text { Relation avec l'oncle } \\
\text { maternel }\end{array}$ & + & + & 0 & 0 & 0 \\
$\begin{array}{l}\text { Aide et assistance de } \\
\text { I'oncle maternel }\end{array}$ & + & + & 0 & 0 & 0 \\
\hline
\end{tabular}

Source: Mohammed ALAHIANE, Le mariage chez les Akhssassi, jeux et stratégies. 
Voici un exemple dans le tableau 2.

Dans ce tableau nous constatons que trois cas sur cinq représentent un mariage endogame à cela il faut ajouter la haut fréquence de mariages endogames dans les milieux aristocratiques urbains; ce qui signifie à la fin que la place que peut jouer la femme dans le processus du développement au Maghreb est déterminée par un ensemble complexe d'éléments. L'élément culturel, politique, économique et religieux et que la libération de celle-ci doit nécessairement passer par la libération de l'homme lui-même, c'est-à-dire de le libérer de ses complexes à l'égard de la femme et de sa pensée traditionnelle et mythologique.

\section{Conclusion}

En guise de conclusion nous n'efforcerons pas à résumer ce qui a été évoqué au long de cet essai. Toutefois, il importe de synthétiser les questions les plus ardues pour mieux cerner la problématique exposée ici.

Dans beaucoup de pays du tiers-monde, quoique le retard technologique et l'influence d'écosystème affectent considérablement le processus du développement. La variante socio-culturelle ne doit pas être négligée dans les analyses des "développeurs"; par ceux-ci on entend les économistes dans leurs bureaux, les politiciens dans les forums officiels, mais aussi et surtout, les banquiers des institutions financières internationales qui, depuis New-York, dressent des programmes d'ajustement pour "sauver" les pays du Sud.

On a pu constater que l'énergie des femmes au Maghreb, comme dans beaucoup de pays arabes, est sous-exploitée à cause de l'influence de la culture. Ainsi ces pays n'arrivent-ils pas à répondre aux besoins d'une population galopante, une situation favorisée par le taux très élevé d'analphabétisme, notamment dans le rang des femmes, et la domination d'une pensée mythique traditionnelle qui empêche une réelle émancipation des deux sexes et un épanouissement de la femme dans la société.

Devant cet état de chose et devant les différents défis que nous impose notre époque, il n'est guère possible de laisser l'effort de toute une partie de la population à l'ombre. Notre pensée et notre culture doivent être actualisées de façon à permettre à tous les individus de se révéler si l'on ne veut pas être oublié par l'histoire. 
1. Une chercheuse américaine écrit: "while Islam has certainly affected the life style of adherent of both sexes this constant cultural factor is insufficient explanation for the considerable range of varation in national levels of female participation within the muslim region".

Susan E. MARSHALL. Economic Development and Culturel Change, University of Chicago, avril 1984, p.499

2. Voir notamment le rôle de son épouse Aïcha avant et après la mort du prophète de l'Islam chez Fatima MERNISSI. Le Harem Politique, Albin Michel, 1987, 294 p.

3. C'est un cas unique pour l'Arabie Saoudite, mais il est important comme exemple.

4. A. MOLAY RCHID, La condition de la femme au Maroc, Rabat 1985, édition de la Faculté des Sciences Juridiques, Economiques et Sociales, p. 81-82.

5. ibidem

6. Par exemple au Maroc l'article 13 de la nomenclature des droits personnels garantis à la femme le droit à l'éducation et au travail. En Algérie le "Code de la famille" basé sur le Coran ne peut le contredire, tandis qu'en Tunisie les droits et libertés individuelles font partie de la logique de laïcité du pays. Voir Attilio GAUDIO, Renée PELLETIER, Femme d'islam ou le Sexe Interdit, Paris, Denoël/ Gonthier, 1980.

7. A. MOULAY RCHID, op.cit., p. 134-135.

8. Au niveau de l'activité féminine le recensement de 1984 en Tunisie révèle que $85 \%$ des femmes mariées sont inactives tandis que 30 à $40 \%$ des femmes célibataires ou divorcées participent aux activités économiques, ce qui confirme que les conraintes au travail féminin proviennent de la famille conjugale et non pas de la société. Cf. Revue Population (Française), nov. déc. 1988, Institut national d'étude démographique, p. 998.

9. Voir Susan, E. MARSHALL. op.cit, p. 502

10. Revue Note et étude documentaire $n^{\circ} 4878,1989-3$, documentation française, p.55

11. $\underline{\text { Ibidem }}$

12. A. MOLAY RCHID. op.cit., p.40

13. Note et étude documentaire, op.cit., p. 8

14. Voir le rapport de la Banque Mondiale sur le développement dans le monde, 1990.

15. Rapport de la Banque Mondiale sur le développement dans le monde. 
16. Le montant de la dette extérieure de l'Algerie est estimé à 24,850 milliards $\$$ celle du Maroc à 19,923 milliards \$ et celle de la Tunisie à 6,672 milliards \$. Cf. Rapport de la BM 1990.

17. Note et étude documentaire, op.cit., p.11

18. La polygynie et la méthode traditionelle d'un homme pour accroître sa progéniture tandis que le divorce, de ce point de vue, si sa fréquence est plus haute, peut apparaître comme un facteur de fécondité élevé par ce qu'il redistribuait des partenaires inféconds entre eux, mais non nécessairement stériles. Et celà selon l'hyptohèse qui avance que, dans la société arabo-musulmane, le divorce sanctionne souvent une union inféconde. Cf. Revue "Population", op.cit., p. 985.

19. Attilio GAUDIO, op.cit., p. 96

20. Revue Population, op.cit., p. 979.

21. Rapport de la Banque Mondiale sur le développement dans le monde 1988.

22. Note et étude documentaire, op.cit., p. 14

23. Ghita EL KHIYAT-BENNAI, Le Monde Arabe au Féminin, ed. Le Fenec, Casablanca (S.D.D.) p. 252

24. Beaucoup de femmes par crainte de leurs proches ou des impôts, cachent leur profession. Cf. Note et étude documentaire, op.cit., p. 29.

25. Annuaire Statistique du Travail (OIT), 1988.

26. Revue du développement. Fonds des Nations Unies pour la femme, $\mathrm{n}^{\circ} 10$, janvier 1986.

27. Jeune Afrique Magazine, $n^{\circ} 63$, octobre 1989, p. 42.

28. Branslaw MALINOWSKI, Une Théorie scientifique de la culture, collect. Point, 1980, p. 35

29. Ahmed MOATASSIME, Femmes Musulmans entre l'état sauvage et les "cultures civilisées". Revue Tiers Monde, Tomme XXV, PUF, Paris, 1984, p. 140

30. Ahmed MOATASSIME, op.cit., p. 142.

31. Ghita EL KHIAT BENNAI, op.cit., p. 222

32. Ibidem, p. 19

33. Ibidem, p. 20

34. Ahmed MOATASSIME, ibidem, p. 142 
35. On fait allusion au voile, que portent les "soeurs musulmanes": mouvement politique et religieux apparaît en Egypte en 1925 (Cfr. Ghita KHIYAT BENNAI, ibid, p. 21) et dans les milieux maghrébins, et Tehador Iranien signe de l'idéologie chéite et de la révolution iranienne.

36. Abdelwahab BOUHDIBA, La sexualité en Islam, Quadroge/PUF, 3e édition, 1982, p. 285.

37. Le Coran cite: "Les femmes sont votre champ. Cultivez-le comme il vous plaira": Cf. Attillio GAUDIO, op.cit., p. 24.

38. Voir différents sens qu'accorde à ce mot le docteur Nawal ESSAADAWI: La face cachée d'Eve La femme dans le monde arabe, Paris, Edition des femmes, 1982, p. 275-277.

39. Jeune Afrique Magazine, $n^{\circ} 48$, mars 1988, p. 33.

40. Beaucoup d'assassinats de femmes mariées sont commis dans tous les pays du Maghreb just après la nuit de noces, ainsi que des divorces et des desocialisation de jeunes filles. Cf. Ghita EL KHIATE BENNAI, op.cit., p. 46, 47. Voir aussi Attilio GAUDIO, op.cit., p. 33.

41. Nawal ESSAADAWI, op.cit., p. 83, voir aussi A. GAUDIO, op.cit., p. 33, il affirme qu'une femme sur deux n'a pas d'hymen.

42. A. GAUDIO, op.cit., p. 115.

43. Exemple au Maroc le Code de statut personnel est très discret sur le prérogatives du mari en cas de non virginité de la femme. (Cfr. A. GAUDIO, op.cit., p. 127), mais le remboursement des biens matrimoniaux, dans de cas, est la forme la plus courante et approuvée par les tribunaux.

44. Ghita EL KHIYAT BENNAI, op.cit, p.60-61. 